\title{
The Relationship between Depression and Insomnia Symptoms Amongst a Sample of UK University Students
}

\author{
Umair Akram, $\mathrm{PhD}^{1,2}$, Asha Akram, $\mathrm{PhD}^{3}$, Maria Gardani, $\mathrm{PhD}^{4}$, Antonia Ypsilanti, $\mathrm{PhD}^{1}$, \\ Kristofor McCarty, $\mathrm{PhD}^{5}$, Sarah Allen, $\mathrm{PhD}^{6}$, Lambros Lazuras, $\mathrm{PhD}^{1}$ \\ 'Department of Psychology, Sociology and Politics, Sheffield Hallam University, Sheffield, UK \\ ${ }^{2}$ Nuffield Department of Clinical Neurosciences, University of Oxford, Oxford, UK \\ ${ }^{3}$ Department of Psychology, The University of Sheffield, Sheffield, UK \\ ${ }^{4}$ School of Psychology, University of Glasgow, Glasgow, UK \\ ${ }^{5}$ Department of Psychology, Faculty of Health and Life Sciences, Northumbria University, Newcastle upon Tyne, UK \\ ${ }^{6}$ Department of Health Sciences, University of York, York, UK
}

Received: March 3, 2019
Revised: April 8, 2019
Accepted: April 19, 2019
Correspondence
Umair Akram, PhD
Department of Psychology,
Sociology and Politics,
Sheffield Hallam University,
Collegiate Crescent, Sheffield,
South Yorkshire, S10 2BP, UK
Tel +44-114-225-3621
E-mail u.akram@shu.ac.uk
ORCID
Umair Akram
https://orcid.org/0000-0003-0150-9274
Asha Akram
https://orcid.org/0000-0002-2079-7761
Maria Gardani
https://orcid.org/0000-0003-0975-5971
Antonia Ypsilanti
https://orcid.org/0000-0003-1379-6215
Kristofor McCarty
https://orcid.org/0000-0001-9189-1520
Sarah Allen
https://orcid.org/0000-0003-0274-4137
Lambros Lazarus
https://orcid.org/0000-0002-5075-9029
Research exploring the relationship between mental health difficulties and insomnia amongst students in the UK remains limited. This study further examined the extent of potential relationships between insomnia, anxiety and depression symptoms amongst university students in the UK. A sample of 487 students from four northern universities completed The Insomnia Severity Index, SLEEP-50, and Hospital Anxiety and Depression Scales. Approximately one fifth of students experienced mild to severe levels of depressive symptoms, and two thirds reported anxious symptoms. One quarter of students indicated insomnia symptoms in the clinical range, however the prevalence of sleep-disorders other than insomnia remained low $(<5 \%)$. Finally, symptoms of insomnia and apnoea were significantly related to depression, after controlling for co-morbid anxiety. The current outcomes highlight the high prevalence of depressive and insomnia symptoms amongst university students in the UK. Considering the relationship between insomnia and depression, student-focused mental health services should explore the possibility of treating insomnia to circumvent depression.

Sleep Med Res 2019;10(1):49-53

Key Words Anxiety, Depression, Insomnia, Sleep, Students mental health.

\section{INTRODUCTION}

Depression is a highly prevalent psychiatric disorder amongst university students affecting up to $31 \%$ of the student population at disorder level [1]. Characterized by lower positive affect, depression leads to impairment in social, occupational and inter-personal function, often manifesting in a number of symptoms including: impaired concentration, fatigue, alteration to weight, hopelessness, and disturbed-sleep [2]. Understanding the co-morbidity between depression and other psychiatric disorders can significantly improve the diagnosis and treatment of depression, potentially allowing for early intervention. Insomnia is arguably the most commonly comorbid disorder with depression (for a review see: [3]), and research concurs that disturbed-sleep amongst students is related to academic, physical and psychological impairment [4]. Indeed, significant life (e.g., leaving home, increased independence) and academic changes (e.g., independent learning) accompany the transition from school/college. For many students, these stressors likely precipitate symptoms of insomnia and mental health difficulties such as depression [5].

Insomnia has recently been recognised as a public health concern, one which can ultimately lead to long-term physical and mental exhaustion, disturbed mood, concentration and memory, and the onset of depression [6-10]. Moreover, meta-analytic results show that in- 
somnia leads to a twofold risk of developing depression [6], and that treating insomnia in those with depression can significantly reduce depressive symptoms [11]. It is well noted that depression often emerges as a consequence of insomnia and anxiety $[6,12]$. Considering poor sleep, anxiety and depression may impair academic performance, students who display such symptoms will certainly be disadvantaged, with limited career opportunities for those falling short of their goals.

To the best of our knowledge only one study has previously examined the relationship between mental health difficulties and insomnia in UK students, specifically from The Oxford Sleep Survey [13]. It emerged that the majority of students clustered into a high-risk psychiatric difficulty group (i.e., displaying symptoms of hallucinations, paranoia, depression, anxiety and hypomania) screened positive for insomnia when compared to those at low-risk. Moreover, amongst the whole sample of students, symptoms of insomnia were most prominently related to anxious and depressive symptoms. Additional research is now required to better understand the interrelationship between symptoms of insomnia, related sleep-disorders, depression and anxiety in the university student population.

The present study aimed to further examine the association between symptoms of insomnia, anxiety and depression amongst the understudied population of university students in the UK. The role of other potentially co-occurring physiological sleepdisorders (e.g., sleep apnoea, narcolepsy) were also examined and controlled for when considering this relationship. Specifically, this study sought to determine: 1) the prevalence of insomnia, anxiety and depression symptoms; 2) the reporting of other sleep disorder symptoms including apnoea, sleepwalking, narcolepsy, restless legs syndrome/periodic limb movement, and circadian rhythm disorder; and 3) the relationship between depression and insomnia symptomology, after controlling for the effects of physiological sleep-disorder and anxious symptoms.

\section{METHODS}

\section{Sample and Procedure}

A cross-sectional online questionnaire-based study was implemented comprising of questions designed to assess the symptoms of depression, anxiety, insomnia and other various sleepdisturbances. The study protocol was approved by the (Masked for Review) University Research Ethics Committee (Protocol number: AMRKT/D\&S-PSP-133), and all participants provided informed consent prior to participation. The survey was advertised to students at four northern universities in the UK through each institutions course credit scheme. 579 participants began the survey, and 478 respondents (mean age $=20.60 \pm 7.72$, range 18-60, 79\% female; $87.7 \%$ undergraduate) who provided complete data were entered into the analysis.

\section{Measures}

Symptoms of anxiety and depression were assessed using the original version of The Hospital Anxiety and Depression Scale (HADS) [14], consisting of 14 items (seven for both the anxiety and depression subscales) scored between 0 and 3, with a maximum score of 21 on both subscales. Higher scores on each subscale represent greater anxiety and depression. For each subscale, a score between: 8-10 indicates mild; $11-14$ moderate; and 15-21 severe symptom severity. Whereas $\leq 7$ indicates a normal (non-case) score. Both subscales demonstrated good internal consistency (Cronbach's $\alpha$ of 0.87 for anxiety, and 0.76 for depression).

Insomnia symptoms were assessed using The Insomnia Severity Index (ISI) [15]. The ISI consists of 7 items examining the severity of insomnia symptoms over the past two weeks including difficulty initiating and maintaining sleep, and awakening too early. Items are scored on a 5-point Likert scale, with total scores ranging from $0-28$. Higher scores represent greater insomnia severity. Specifically, a score between: $0-7$ indicates no clinically significant insomnia; 8-14 subthreshold insomnia; 15-21 clinical insomnia of moderate severity; and 22-28 severe clinical insomnia. Assessment of internal consistency yielded a Cronbach's alpha of 0.86 .

Subscales of the SLEEP-50 [16] examined symptoms of apnoea, sleepwalking, narcolepsy, restless-legs syndrome/periodic limb-movement (RLS/PLMD), and circadian-rhythm disorder (CRD). The SLEEP-50 consists of fifty items; in the current study items relating to insomnia (assessed by the ISI) or sleep hygiene were omitted. Participants were asked to rate to what extent the items have been applicable in the past month $(0=$ not at all, 4 = very much). An example item is: "I am told that I wake up gasping for air." Higher scores indicate a greater presence of the specified symptom. Total scores of: $\geq 15$ indicate apnea; $\geq 7$ sleepwalking; $\geq 7$ narcolepsy; $\geq 7$ restless legs syndrome/periodic limb movement; and $\geq 8$ a circadian rhythm disorder. Assessment of internal consistency yielded a Cronbach's alpha of: 0.70 for apnea; 0.61 for sleepwalking; 0.64 for narcolepsy; 0.68 for restless legs syndrome/periodic limb movement; and 0.60 for a circadian rhythm disorder.

\section{RESULTS}

\section{Prevalence}

Mean scores for the final sample were as follows: ISI, $10.43 \pm$ 5.96; anxiety, $8.93 \pm 4.64$; depression, $4.84 \pm 3.46$; apnoea, 4.52 \pm 3.32; narcolepsy, $2.31 \pm 2.33$; RLS/PLMD, $2.03 \pm 2.32$; CRD, $2.60 \pm 2.18$; sleepwalking, $0.57 \pm 1.24$.

The results demonstrated that $39.1 \%$ of students indicated subthreshold insomnia and $25.3 \%$ were in the clinical range ( $21.3 \%$ moderate, $4 \%$ severe). The prevalence of anxiety symptoms was rather high with $22.6 \%$ of the sample reporting bor- 
derline levels and $35.8 \%$ presenting in the clinical range. Whereas, depressive symptoms were somewhat lower with $12.6 \%$ of students reporting borderline levels and 6.3\% reporting clinical levels. Finally, measurement of sleep characteristics showed that: $1.3 \%$ indicated apnoea symptoms; $5 \%$ narcolepsy symptoms; $4.6 \%$ RLS/PLMD; 3.1\% a CRD; and no instances of sleepwalking. Independent samples t-tests demonstrated that reports of RLS/PLMD symptoms were significantly higher amongst females $(\mathrm{M}=2.15 \pm 2.07)$ when compared to males $(\mathrm{M}=1.61 \pm$ 2.07: $\mathrm{t}(485)=-2.09, \mathrm{p}=0.04)$. No other sex differences were observed. Correlations are presented in Table 1.

\section{Relationship between Insomnia and Depressive Symptoms}

A bootstrapped (2000 resamples) hierarchical linear regression analysis assessed the association between sleep-disturbances, insomnia and depressive symptoms after controlling for anxiety, age and sex. This was completed in two steps and the overall model predicted $48.7 \%$ (adjusted $\mathrm{R}^{2}$ ) in depression, a large multivariate effect size $\left(\mathrm{f}^{2}=0.94\right)$ according to Cohen et al. [17]. The Mahalanobis Distance test identified nine cases as having a high probability of being an outlier $(\mathrm{p}=0.001)$ and were removed from the dataset. We also inspected tolerance levels and the lowest score was 0.618 , therefore indicating low multicollinearity among predictor variables.

Table 1. Correlations for anxiety, depression, insomnia symptoms, and SLEEP-50 scores

\begin{tabular}{|c|c|c|c|c|c|c|c|}
\hline & 1. & 2. & 3. & 4. & 5. & 6. & 7. \\
\hline \multicolumn{8}{|l|}{ 1. Insomnia } \\
\hline 2. Anxiety & $0.51^{*}$ & & & & & & \\
\hline 3. Depression & $0.53^{*}$ & $0.66^{*}$ & & & & & \\
\hline 4. Apnoea & $0.33^{*}$ & $0.33^{*}$ & $0.36^{*}$ & & & & \\
\hline 5. Narcolepsy & $0.41^{*}$ & $0.34^{*}$ & $0.35^{*}$ & $0.52^{*}$ & & & \\
\hline 6. RLS/PLMD & $0.26^{*}$ & $0.31^{*}$ & $0.29^{*}$ & $0.40^{*}$ & $0.38^{*}$ & & \\
\hline 7. CRD & $0.45^{*}$ & $0.33^{*}$ & $0.30^{*}$ & $0.32^{*}$ & $0.37^{*}$ & $0.29^{*}$ & \\
\hline 8. Sleepwalking & 0.08 & 0.08 & 0.08 & $0.27^{*}$ & $0.22^{*}$ & $0.16^{*}$ & $0.17^{*}$ \\
\hline
\end{tabular}

${ }^{*} \mathrm{p}<0.001$. RLS/PLM: restless legs syndrome/periodic limb movement, CRD: circadian rhythm disorder.

Table 2. Linear regression analyses with depression as the dependant variable and age, sex, SLEEP-50 scores and anxiety as predictors

\begin{tabular}{|c|c|c|c|c|}
\hline & B & $\beta$ & $95 \%$ CIs for B & $\mathrm{R}^{2}$ \\
\hline Step 1 & & & & $33.4 \%$ \\
\hline Age & 0.040 & 0.058 & $-0.013-0.094$ & \\
\hline Sex & -0.165 & -0.020 & $-0.802-0.471$ & \\
\hline Sleep apnoea & 0.176 & $0.154^{* *}$ & $0.073-0.279$ & \\
\hline Narcolepsy & 0.108 & 0.068 & $-0.035-0.250$ & \\
\hline RLS/PLM & 0.149 & $0.097^{*}$ & $0.017-0.281$ & \\
\hline CRD & -0.016 & -0.010 & $-0.156-0.125$ & \\
\hline Sleepwalking & -0.163 & -0.047 & $-0.429-0.104$ & \\
\hline Insomnia & 0.252 & $0.434^{* * *}$ & $0.200-0.303$ & \\
\hline Step 2 & & & & $49.5 \%$ \\
\hline Age & 0.033 & 0.047 & $-0.013-0.079$ & \\
\hline Sex & -0.319 & -0.038 & $-0.871-0.234$ & \\
\hline Sleep apnoea & 0.099 & $0.087^{*}$ & $0.009-0.189$ & \\
\hline Narcolepsy & 0.036 & 0.022 & $-0.089-0.160$ & \\
\hline RLS/PLM & 0.044 & 0.029 & $-0.072-0.160$ & \\
\hline CRD & -0.054 & -0.034 & $-0.176-0.068$ & \\
\hline Sleepwalking & -0.130 & -0.038 & $-0.361-0.101$ & \\
\hline Insomnia & 0.133 & $0.229^{* * *}$ & $0.085-0.181$ & \\
\hline Anxiety & 0.376 & $0.510^{* * *}$ & $0.317-0.436$ & \\
\hline
\end{tabular}

${ }^{*} \mathrm{p}<0.05 .^{* *} \mathrm{p}<0.005 .{ }^{* * *} \mathrm{p}<0.001$.

RLS/PLM: restless legs syndrome/periodic limb movement, CRD: circadian rhythm disorder. 
The first step of the model included demographic characteristics (age and sex); sleep problems (i.e., symptoms of: sleep apnoea, narcolepsy, restless legs syndrome/periodic limb movement, circadian rhythm disorder, and sleepwalking); and insomnia, as measured by the ISI, and predicted $31.6 \%$ (adjusted $\mathrm{R}^{2}$ ) of the variance in depressive symptoms. In the first step of the analysis, depressive symptoms were positively associated with RLS/PLM symptoms $(\beta=0.097, \mathrm{p}=0.028)$, sleep apnoea symptoms $(\beta=$ $0.154, \mathrm{p}=0.001)$, and ISI scores $(\beta=0.434, \mathrm{p}=0.001)$. To better understand the association between sleep problems and insomnia with depressive symptoms, and given the high co-morbidity between depression and anxiety, we statistically controlled for the effects of anxiety (HADS-D) in the second step of the analysis. Adding HADS-A scores in the second step significantly increased the predicted variance in depressive symptoms by $16.9 \%$ $\left(\mathrm{F}_{\text {change }}=152.62, \mathrm{p}=0.001\right)$, and there was a strong positive association between anxiety and depressive symptoms $(\beta=0.510$, $\mathrm{p}=0.001$ ). The relationships between sleep apnoea symptoms and insomnia with depression were retained. However, the relationship between symptoms of restless legs syndrome/periodic limb movement and depression became non-significant (Table 2).

\section{DISCUSSION}

Young adulthood is a critical time period for the onset of insomnia and depression, particularly in the student population [18]. Despite this, research exploring the relationship between these two psychiatric difficulties amongst students in the UK remains limited. The results from our study indicated that approximately one fifth of students in the current sample experienced mild to severe levels of depressive symptoms, and two thirds reported anxious symptoms. Moreover, whilst one quarter indicated insomnia symptoms in the clinical range, the prevalence of sleep-disorders other than insomnia remained low (< $5 \%$ ). These prevalence rates are comparable with previous research reporting high levels of depressive and/or insomnia symptoms in American university students and [19,20] appear to be markedly higher than point prevalence rates established amongst the general population $[21,22]$.

The present study also demonstrated that symptoms of insomnia and apnoea were significantly related to depression, after controlling for co-morbid anxiety which is commonly related to both difficulties $[6,7,12]$. Symptoms of anxiety may perpetuate insomnia due to increased worry and ruminative thinking when attempting to initiate sleep [23], and among students this may relate to the stress of academic life. The nature of this thought pattern may lead to negatively toned cognitive activity, arousal and distress which delays sleep-onset [24]. In turn, daytime consequences of insomnia and/or apnoea may impair the ability to adequately cope with social and interpersonal difficulties and academic stressors faced by stu- dents, which may precipitate symptoms of depression [25].

Distress and low wellbeing among young adults is rising [1] with a subsequent rise in instances of poor wellbeing and distress amongst university students. Recent reports show an alarming approx. Fivefold increase in the number of students who disclose a mental health condition to their institution over the past decade. Consequently, student counselling and disability services have fell short when meeting the demands for support required [26]. Considering that the consequences of depression can be severe for the academic, social, and professional lives of university students [17] student-focused mental health services in the UK need to improve screening processes to identify at-risk populations and engage in early intervention [27]. Indeed, psychiatric difficulties can be easily identified and targeted for intervention amongst young adults [13] and screening for sleep-disorders such as insomnia would certainly provide a useful advancement considering its preceding relationship with depression [12].

Several limitations should be noted. The students in the current sample were recruited from only four universities where they were remunerated with course credit. Moreover, the present sample consisted mostly of female participants, and as such the present findings may not be fully generalizable to males and all UK university students. However, it is relevant to note that women are more likely than men to experience both insomnia and depression [28,29]. Moreover, as the mean age of the current sample was relatively young, the outcomes may not extend to older adults who are perusing a university education. Next, considering the cross-sectional nature of the current data, we cannot ascertain whether there is an increase in new onset depression associated with student life or a potential effect rolled over by adolescent sleep and depressive difficulties. Finally, whilst symptomology of sleep disorders other than insomnia were subjectively examined (e.g. apnoea, narcolepsy), objective assessments of sleep (i.e. actigraphy, polysomnography) should be used to confirm diagnosis. With these caveats in mind, future, more rigorous research should confirm the current outcomes amongst a more representative and balanced sample.

In sum, the current outcomes highlight the high prevalence of depressive and insomnia symptoms amongst university students in the UK. Moving forward, research should consider the role of underlying psychological and psychosocial factors that may serve to explain this relationship. Finally, considering the efficacy of reducing depressive symptoms through the treatment of insomnia [11], further research should also examine the efficacy of institutional student sleep interventions in circumventing depression.

\section{Conflicts of Interest}

The authors have no financial conflicts of interest.

\section{Authors' Contribution}

This study was designed and conceived by UA. Data was analysed by LL 
and UA. An initial version of this manuscript was written by UA and LL. Following, input was sought from all other authors. All authors approved the final version of this manuscript.

\section{REFERENCES}

1. Ibrahim AK, Kelly SJ, Adams CE, Glazebrook C. A systematic review of studies of depression prevalence in university students. J Psychiatr Res 2013;47:391-400.

2. American Psychiatric Association. Diagnostic and Statistical Manual of Mental Disorders ( $5^{\text {th }}$ ed. DSM-5). Washington, DC: American Psychiatric Pub 2013.

3. Riemann D, Voderholzer U. Primary insomnia: a risk factor to develop depression? J Affect Disord 2003;76:255-9.

4. Hershner SD, Chervin RD. Causes and consequences of sleepiness among college students. Nat Sci Sleep 2014;6:73-84.

5. Taylor DJ, Vatthauer KE, Bramoweth AD, Ruggero C, Roane B. The role of sleep in predicting college academic performance: is it a unique predictor? Behav Sleep Med 2013;11:159-72.

6. Baglioni C, Battagliese G, Feige B, Spiegelhalder K, Nissen C, Voderholzer $\mathrm{U}$, et al. Insomnia as a predictor of depression: a meta-analytic evaluation of longitudinal epidemiological studies. J Affect Disord 2011;135:10-9.

7. Choueiry N, Salamoun T, Jabbour H, El Osta N, Hajj A, Rabbaa Khabbaz L. Insomnia and relationship with anxiety in university students: a cross-sectional designed study. PLoS One 2016;11:e0149643.

8. Ellis JG, Perlis ML, Bastien CH, Gardani M, Espie CA. The natural history of insomnia: acute insomnia and first-onset depression. Sleep 2014;37:97-106.

9. Kyle SD, Morgan K, Espie CA. Insomnia and health-related quality of life. Sleep Med Rev 2010;14:69-82.

10. Perlis ML, Giles DE, Buysse DJ, Thase ME, Tu X, Kupfer DJ. Which depressive symptoms are related to which sleep electroencephalographic variables? Biol Psychiatry 1997;42:904-13.

11. Manber R, Edinger JD, Gress JL, San Pedro-Salcedo MG, Kuo TF, Kalista T. Cognitive behavioral therapy for insomnia enhances depression outcome in patients with comorbid major depressive disorder and insomnia. Sleep 2008;31:489-95.

12. Ford DE, Kamerow DB. Epidemiologic study of sleep disturbances and psychiatric disorders. An opportunity for prevention? JAMA 1989; 262:1479-84.

13. Sheaves B, Porcheret K, Tsanas A, Espie CA, Foster RG, Freeman D, et al. Insomnia, nightmares, and chronotype as markers of risk for severe mental illness: results from a student population. Sleep 2016;39:173-81.

14. Zigmond AS, Snaith RP. The hospital anxiety and depression scale. Acta Psychiatr Scand 1983;67:361-70.

15. Bastien CH, Vallières A, Morin CM. Validation of the Insomnia Severity Index as an outcome measure for insomnia research. Sleep Med 2001;2:297-307.

16. Spoormaker VI, Verbeek I, van den Bout J, Klip EC. Initial validation of the SLEEP-50 questionnaire. Behav Sleep Med 2005;3:227-46.

17. Cohen AA, Milot E, Yong J, Seplaki CL, Fülöp T, Bandeen-Roche K, et al. A novel statistical approach shows evidence for multi-system physiological dysregulation during aging. Mech Ageing Dev 2013;134:110-7.

18. Kessler RC, Zhao S, Blazer DG, Swartz M. Prevalence, correlates, and course of minor depression and major depression in the National Comorbidity Survey. J Affect Disord 1997;45:19-30.

19. Gress-Smith JL, Roubinov DS, Andreotti C, Compas BE, Luecken LJ. Prevalence, severity and risk factors for depressive symptoms and insomnia in college undergraduates. Stress Health 2015;31:63-70.

20. Lund HG, Reider BD, Whiting AB, Prichard JR. Sleep patterns and predictors of disturbed sleep in a large population of college students. $J$ Adolesc Health 2010;46:124-32.

21. Sun X, Zheng B, Lv J, Guo Y, Bian Z, Yang L, et al. Sleep behavior and depression: findings from the China Kadoorie Biobank of 0.5 million Chinese adults. J Affect Disord 2018;229:120-4.

22. Ohayon MM. Epidemiology of insomnia: what we know and what we still need to learn. Sleep Med Rev 2002;6:97-111.

23. Akram U, Ellis JG, Barclay NL. Anxiety mediates the relationship between perfectionism and insomnia symptoms: a longitudinal study. PLoS One 2015;10:e0138865.

24. Harvey AG. A cognitive model of insomnia. Behav Res Ther 2002;40: 869-93.

25. Staner L. Comorbidity of insomnia and depression. Sleep Med Rev 2010;14:35-46.

26. Thorley C. Not By Degrees: improving student mental health in the UK's universities. London: Institute for Public Policy Research 2017.

27. Hill RM, Yaroslavsky I, Pettit JW. Enhancing depression screening to identify college students at risk for persistent depressive symptoms. $J$ Affect Disord 2015;174:1-6.

28. Nolen-Hoeksema S. Gender differences in depression. Curr Dir Psychol Sci 2001;10:173-6.

29. Zhang B, Wing YK. Sex differences in insomnia: a meta-analysis. Sleep 2006;29:85-93. 